\title{
Leserbrief
}

\section{Diskurs zur anti-inflammatorischen Therapie des Adenoid-Syndroms}

\author{
Zum CME-Beitrag „Diagnostik und Therapie des Adenoid- \\ Syndroms", den wir in der letzten Ausgabe veröffentlicht \\ haben, erreichte uns der im folgenden abgedruckte Leserbrief \\ von Herrn Prof. Dr. Boris A. Stuck, zu dem die Autoren des \\ Beitrags in Anschluss Stellung nehmen. Die jeweils angeführte \\ Literatur liegt dem Verlag vor.
}

Im ihrem Artikel „Diagnostik und Therapie des Adenoid-Syndroms - Alternative Operationsverfahren nur im Ausnahmefall “ erläutern die Autoren die aktuelle Diagnostik und Therapie der hyperplastischen kindlichen Adenoide. Die Autoren schildern sehr zutreffend die klinische Symptomatik, beziehen sich erfreulicherweise auf die gängigen interdisziplinären Empfehlungen zur präoperativen Gerinnungsdiagnostik und legen einen Schwerpunkt auf die operative Therapie einschließlich der möglichen Komplikationen und des postoperativen Verlaufs. Sicher auch aufgrund des operativen Schwerpunktes des Artikels äußern sich die Autoren nur knapp zu den konservativen Therapieoptionen und kommen hierbei zum Schluss: „Zur Überlegenheit einer rein konservativen Therapie liegen keine evidenzbasierten Studien vor" sowie „eine prätherapeutische Therapie mit topischen Kortikosteroiden konnte bisher keinen Nutzen nachweisen".

An dieser Stelle möchte ich mir erlauben, den insgesamt sehr gelungenen Artikel zu ergänzen.

Insbesondere in der Therapie des kindlichen Schnarchens und der kindlichen obstruktiven Schlafapnoe wurden in den letzten Jahren zahlreiche methodisch anspruchsvolle Studien publiziert, die die Wirksamkeit der konservativen Therapie mit anti-inflamma-torischen Medikamenten untermauern. Insbesondere belegen mehrere teils randomisierte, placebokontrollierte Studie die Wirksamkeit der topischen Steroide bei der adenoiden Hyperplasie [1-9]. Darüber hinaus wurden in den letzten Jahren vermehrt Arbeiten zur Wirksamkeit einer systemischen anti-inflammatorischen Therapie mit Leukotrien-Antagonisten publiziert [9-12].

Beispielhaft konnten Kheirandish-Gozal et al. 2008 in einer randomisierten, placebokontrollierten Cross-over-Studie an 62 Kindern zeigen, dass sich durch intranasale Steroide die Schwere der obstruktiven Schlafapnoe signifikant verbessern ließ [7]. Eine Verbesserung der subjektiven Symptome und eine Reduktion der Adenoide ließ sich auch für Fluticason Nasentropfen anhand einer placebokontrollierten randomisierten Studie von Demirhan et al. dokumentieren [6]. In der aktuellsten Arbeit von Goldbart et al. aus dem Jahr 2012 konnte in einer randomisierten, placebokontrollierten Studie an 46 Kindern mit obstruktiver Schlafapnoe eine Überlegenheit gegenüber Placebo und eine signifikante Reduktion

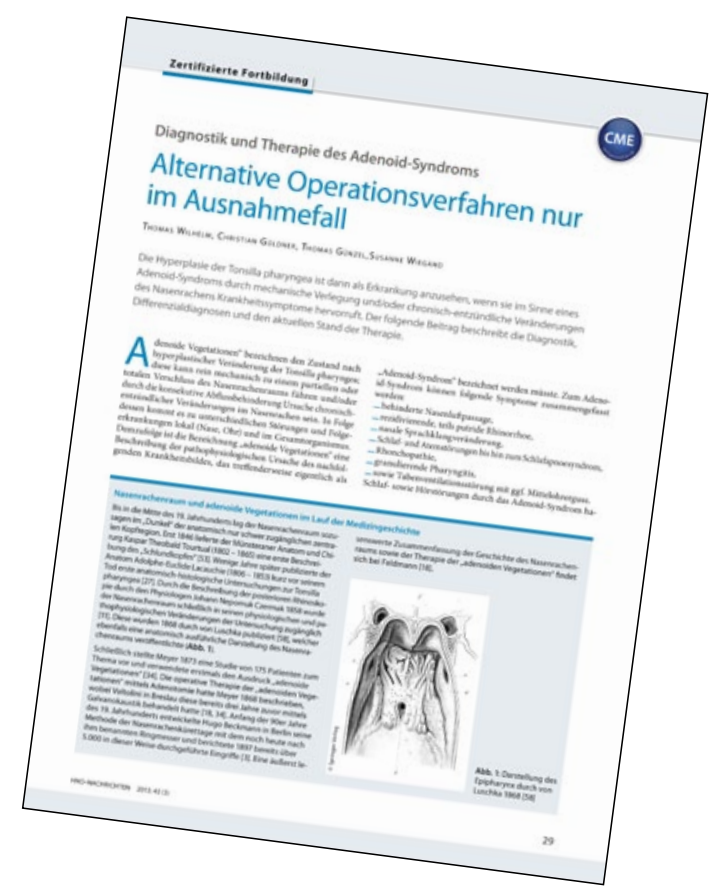

der Schwere der Atmungsstörung durch Montelukast dokumentiert werden [11]. In den genannten Arbeiten ließ sich eine weitgehende Remission der Beschwerden in einem hohen Prozentsatz der Patienten erreichen (auf eine zunächst geplante operative Entfernung der Adenoide konnte z.B. in der Arbeit von Demirhan et al. in $76 \%$ der mit topischen Steroiden behandelten Kinder verzichtet werden). Die Therapie mit anti-inflammatorischen Medikamenten erwies sich für die verwendeten Präparate als sehr gut verträglich. Anti-inflammatorische Medikamente eröffnen auch auf dem Boden der eigenen klinischen Erfahrung eine wirksame therapeutische Alternative bei Kindern mit einer symptomatischen Hyperplasie der Adenoide. Auch wenn sicher nicht alle Kinder mit dieser Therapie alleine ausreichend behandelt werden können und noch einige offene Fragen zum Langzeitverlauf bestehen, so sollte diese Therapieoption den betroffenen Kindern nicht vorenthalten werden. Die Indikation zur operativen Therapie der Adenoide wird immer individuell getroffen. Gerade in den Fällen, in denen die Indikation fraglich ist oder die Eltern einer operativen Therapie ablehnend gegenüberstehen, sollte die Möglichkeit der konservativen Therapie in Betracht gezogen werden.

Prof. Dr. med. Boris A. Stuck

Universitäts-HNO-Klinik Mannheim, Theodor-Kulzer-Ufer 1-3

68167 Mannheim 